\title{
Effect of Surface Finish on the Failure Mechanisms of Flip-Chip Solder Joints Under Electromigration
}

\author{
Y.L. LIN, ${ }^{1}$ Y.S. LAI, ${ }^{2}$ C.M. TSAI, ${ }^{1}$ and C.R. $\mathrm{KAO}^{3}$ \\ 1.-Department of Chemical \& Materials Engineering, National Central University, Jhongli City, \\ Taiwan, Republic of China. 2.-Advanced Semiconductor Engineering, Inc., Kaohsiung City, \\ Taiwan, Republic of China. 3.-Department of Materials Science \& Engineering, National \\ Taiwan University, Taipei, Taiwan, Republic of China; e-mail: kaocr@hotmail.com
}

Two substrate surface finishes, $\mathrm{Au} / \mathrm{Ni}$ and organic solderable preservative (OSP), were used to study the effect of the surface finish on the reliability of flip-chip solder joints under electromigration at $150^{\circ} \mathrm{C}$ ambient temperature. The solder used was eutectic PbSn, and the applied current density was $5 \times$ $10^{3} \mathrm{~A} / \mathrm{cm}^{2}$ at the contact window of the chip. The under bump metallurgy (UBM) on the chip was sputtered $\mathrm{Cu} / \mathrm{Ni}$. It was found that the mean-timeto-failure (MTTF) of the OSP joints was six times better than that of the $\mathrm{Au} / \mathrm{Ni}$ joints $(3080 \mathrm{~h}$ vs. $500 \mathrm{~h}$ ). Microstructure examinations uncovered that the combined effect of current crowding and the accompanying local Joule heating accelerated the local Ni UBM consumption near the point of electron entrance. Once $\mathrm{Ni}$ was depleted at a certain region, this region became nonconductive, and the flow of the electrons was diverted to the neighboring region. This neighboring region then became the place where electrons entered the joint, and the local Ni UBM consumption was accelerated. This process repeated itself, and the Ni-depleted region extended further on, creating an ever-larger nonconductive region. The solder joint eventually failed when the nonconductive region became too large, making the effective current density very high. Accordingly, the key factor determining the MTTF was the Ni consumption rate. The joints with the OSP surface finish had a longer MTTF because $\mathrm{Cu}$ released from the substrate was able to reduce the Ni consumption rate.

Key words: Flip-chip, electromigration, solder, failure mechanism.

\section{INTRODUCTION}

The failure of solder joints triggered by high current density is one of the key reliability concerns of the flip-chip devices. ${ }^{1-14}$ To counter the negative effects caused by high current density, several approaches have been proposed and deployed. Two well-known approaches involve better circuit and interconnect designs. The first is to distribute the current load to as many solder joints as possible so that the current carried by each joint becomes smaller. The second is to fine-tune the geometry of the interconnect as well as that of the under bump metallurgy (UBM) so that the high local current density region and the current-crowding region fall on materials that are more resistant to high current density rather than on solders, which are more susceptible to problems caused by high current density.

(Received March 9, 2006; accepted June 29, 2006)
Experimenting with new UBM and surface finish combinations is also an approach that has high potential. In this paper, two different substrate surface finishes, $\mathrm{Au} / \mathrm{Ni}$ and organic solderable preservative $(\mathrm{OSP}) / \mathrm{Cu}$, were used in combination with the $\mathrm{Cu} / \mathrm{Ni} / \mathrm{Al} \mathrm{UBM}$ to study the effect of the surface finish on the reliability of flip-chip solder joints under current stressing. After assembly, the $\mathrm{Au}$ layer of the $\mathrm{Au} / \mathrm{Ni}$ surface finish, the $\mathrm{Cu}$ layer of the $\mathrm{Cu} / \mathrm{Ni} / \mathrm{Al} \mathrm{UBM}$, and the OSP will disappear from the interface, leaving the $\mathrm{Ni}$ or $\mathrm{Cu}$ layers beneath exposed to solder. As a result, the materials sequence for the $\mathrm{Au} / \mathrm{Ni}$ joints and the OSP joints will be $\mathrm{Ni} /$ solder/Ni and $\mathrm{Ni} /$ solder/Cu, respectively. One interesting aspect of the $\mathrm{Ni} / \mathrm{solder} / \mathrm{Cu}$ combination is that $\mathrm{Ni}$ and $\mathrm{Cu}$ can diffuse across the solder to interact with the other element, ${ }^{15,16}$ causing the $\mathrm{Ni}$ consumption rate to decrease and the $\mathrm{Cu}$ consumption rate to increase. It is interesting to see if this cross-interaction will influence the reliability of solder joints under current stressing. 


\section{EXPERIMENTAL}

The test chips had a daisy-chain circuit and were mounted on substrates. The solder joints had the configuration illustrated schematically in Fig. 1. The chips had $\mathrm{Al}$ interconnect lines that were 60 $\mu \mathrm{m}$ wide and $1 \mu \mathrm{m}$ thick. The UBM on the chips had the $\mathrm{Cu} / \mathrm{Ni} / \mathrm{Al}$ structure. The $\mathrm{Cu}$ and the $\mathrm{Ni}$ layers were sputter-deposited and were $0.8 \mu \mathrm{m}$ and $0.5 \mu \mathrm{m}$ thick, respectively. The Ni layer was deposited by sputtering and as a result contained a small amount of vanadium. The diameter of the contact window was $90 \mu \mathrm{m}$. On the substrate side, two different sets of surface finish, $\mathrm{Au} / \mathrm{Ni}$ and OSP were used. The $\mathrm{Au}$ and the Ni layers of the $\mathrm{Au} / \mathrm{Ni}$ were $0.65 \mu \mathrm{m}$ and $5 \mu \mathrm{m}$, respectively. The solder was eutectic $\mathrm{PbSn}$, and the solder joints had a nominal diameter of $125 \mu \mathrm{m}$.

For electromigration testing, we kept the assembled samples in an oven set at $150^{\circ} \mathrm{C}$, and a constant 0.32 A current was applied, producing a nominal current density of $5 \times 10^{3} \mathrm{~A} / \mathrm{cm}^{2}$ (basing on the opening area of the contact window). To monitor the change of temperature, thermal couples were attached on the backsides of each chip at the locations immediately above the $\mathrm{Al}$ interconnect where the electron current passed through. Meanwhile, resistance change was also monitored every $10 \mathrm{~s}$, and the time-to-failure was determined by an abrupt potential increase in the potential-time curve. To calculate the mean-time-to-failure (MTTF), the time-to-failure data from 22 samples were used.

After current stressing at different periods of time, the solder joints were cross-sectioned through the middle of the contact window and examined using a scanning electron microscope (SEM). For samples with the UBM depleted, the structure at the interfacial region between $\mathrm{Al}$ line and UBM was so weak that the focused ion beam (FIB) had to be used to perform final polishing to remove the smeared solder to uncover the features below. The compositions of the intermetallic compounds were determined by electron probe microanalysis (EPMA).

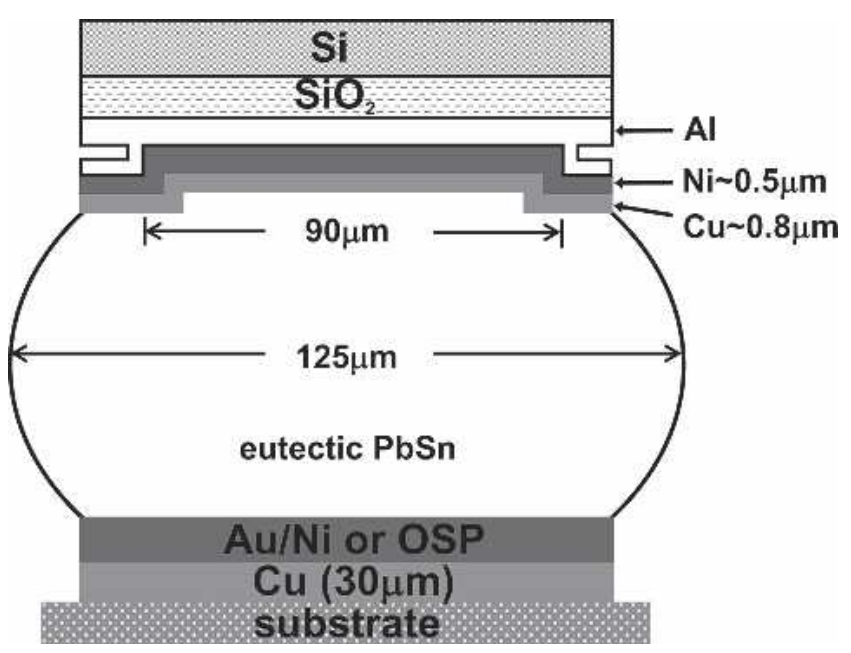

Fig. 1. Schematic drawing showing the solder joint configuration used in this study.

\section{RESULTS}

The MTTF for the solder joints with the Au/Ni surface finish was $530 \mathrm{~h}$, and that with the OSP was almost six times longer at 3,081 h. The MTTF values reported here were based on 22 samples for each surface finish. It was very surprising that the surface finish alone was able to bring on such a huge performance difference, even though everything else was kept the same. In the following, the results from the microstructure examinations at different stages of current stressing are presented to uncover the reasons for this strong surface finish effect.

\section{OSP Surface Finish}

Figure 2 shows the microstructures of the OSP joints at different stages of their microstructure evolution. The electrons entered the joints from the upper-right corners. After $700 \mathrm{~h}$ current stressing, as shown in Fig. 2a, the eutectic PbSn had coarsened substantially due to the high environmental temperature $\left(150^{\circ} \mathrm{C}\right)$. The upper-right corner and the upper-left corner of Fig. $2 \mathrm{a}$ are shown in Figs. $3 \mathrm{a}$ and $3 \mathrm{~b}$, respectively. Figures $3 \mathrm{a}$ and $3 \mathrm{~b}$ show that the original $0.8 \mu \mathrm{m}$ layer of $\mathrm{Cu}$ had been replaced by a series of $\mathrm{Cu}_{6} \mathrm{Sn}_{5}$ scallops. The scallops on the right side of Fig. 3a emitted Ni signals during EPMA analysis, suggesting that these scallops had became $\left(\mathrm{Cu}_{1-\mathrm{x}} \mathrm{Ni}_{\mathrm{x}}\right)_{6} \mathrm{Sn}_{5}$. These two micrographs also show that the Ni UBM on the right side of the joint had been depleted and replaced by a so-called "twophase structure," while most of the Ni UBM on the left side of the joint still remained. This suggests that the Ni consumption rate was not homogeneous and was higher on the side where electrons entered the joint. This two-phase structure had been reported before in the literature but was referred to as the "porous structure," 14 but its exact nature is still unknown. The FIB technique was used to remove the solder smear formed during sample polishing to have a better view of the two-phase structure, as shown in Fig. 4. Even at this magnification, the exact nature of this structure still could not be identified. Transmission electron microscopy is needed to fully characterize it. Nevertheless, it is assumed that this layer was nonconductive and blocked the electron flow through this region.

When the current stressing time reached $1700 \mathrm{~h}$, there was a void at the central region on the chip side of the joint, as shown in Fig. 2b. It is worth noting that the void did not start at the upper-right corner of the joint, where electrons entered the joint initially. This are was believed to have the highest local current density and the highest degree of current crowding initially. ${ }^{2-10}$ Figure 5 is a zoom-in image for the left side of the void shown in Fig. $2 b$. It is worth noting that the Ni UBM above the void was still intact, and the intermetallic above the void was $\mathrm{Cu}_{6} \mathrm{Sn}_{5}$.

When the current stressing time reached $2,700 \mathrm{~h}$, the void had propagated toward the left side along the interface to the outer edge of the joint, as shown in Fig. 2c. After 3,000 h current stressing, the solder 

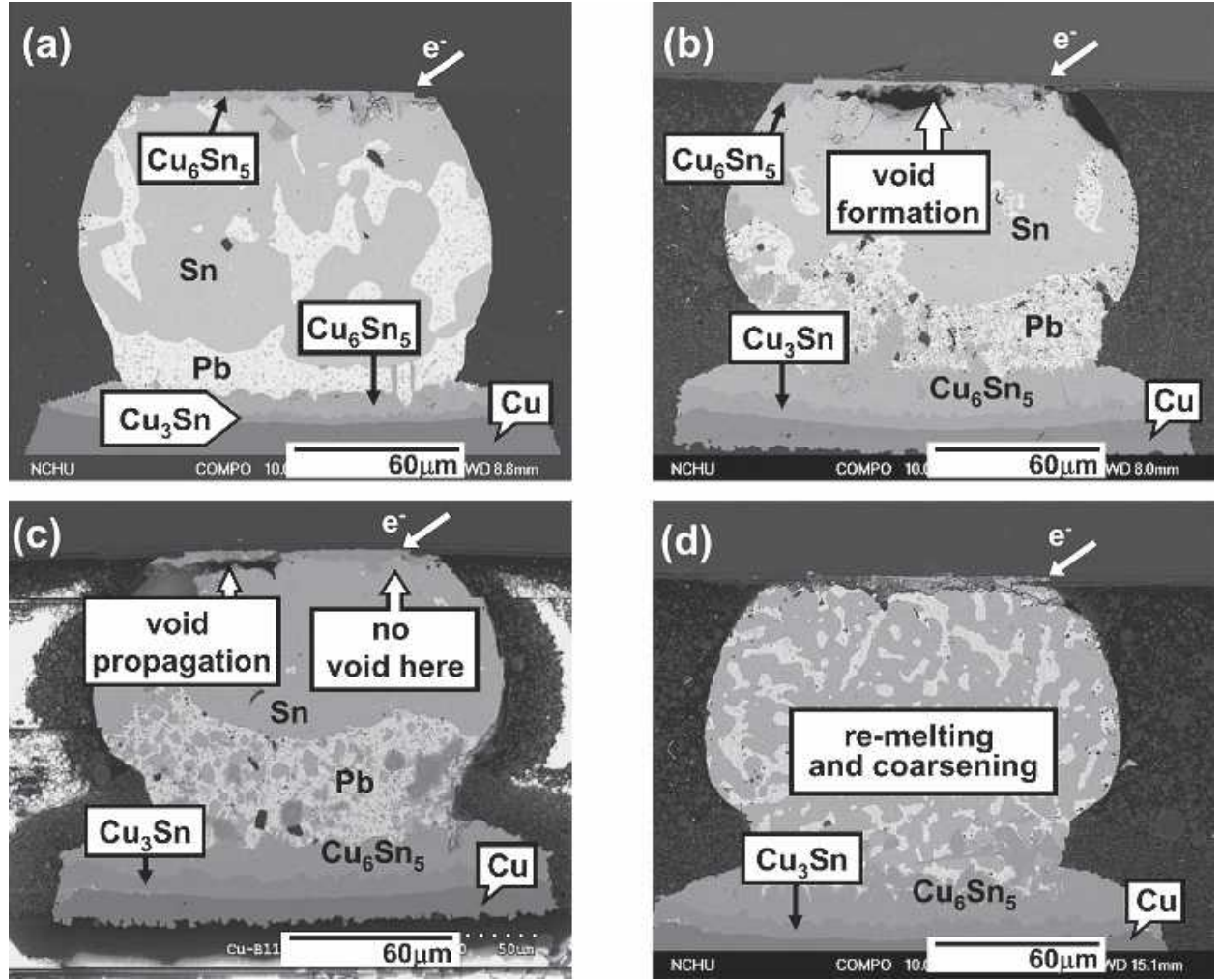

Fig. 2. Microstructures of the OSP joints that had been stressed at $150^{\circ} \mathrm{C}$ for (a) $700 \mathrm{~h}$, (b) $1,700 \mathrm{~h}$, (c) $2,700 \mathrm{~h}$, and (d) $3,000 \mathrm{~h}$. The electrons entered the joints from the upper-right corners.

joint failed, as shown in Fig. 2d. The joint experienced a complete melting, as evidenced by the finegrain eutectic $\mathrm{PbSn}$ structure. At the time of failure, the thermal couple also registered a temperature higher than the eutectic temperature.

Figures 2a-2c also clearly demonstrate the trend that $\mathrm{Pb}$ atoms were driven toward the anode (substrate) side. At 2,700 h, shown in Fig. 2c, the Sn-rich phase had aggregated into a large continuous region near the cathode side. Figures $6 a-6 d$ show the solder joints with the opposite polarity; i.e., electrons entered the joints from the substrates and left the joints through the chip. Again, $\mathrm{Pb}$ atoms were driven toward the anode side, but then the anode side was the chip side. These two observations show that $\mathrm{Pb}$ atoms, which had a higher diffusivity at $150^{\circ} \mathrm{C},{ }^{6,9}$ migrated in the same direction as that of the electrons. This is a positive proof that electromigration was the main driving force for solder migration in this study.

\section{Au/Ni Surface Finish}

Figure 7 shows the microstructures of the $\mathrm{Au} / \mathrm{Ni}$ joints at different stressing time. The test conditions were the same as those for the OSP joints. The microstructure before current stressing is shown in Fig. 7a. The $0.8-\mu \mathrm{m} \mathrm{Cu}$ layer of the UBM had completely been replaced by $\mathrm{Cu}_{6} \mathrm{Sn}_{5}$, and the $\mathrm{Au}$ layer of the surface finish had been replaced by $\left(\mathrm{Cu}_{1-\mathrm{x}} \mathrm{Ni}_{\mathrm{x}}\right)_{6} \mathrm{Sn}_{5}$. In other words, the $0.8-\mu \mathrm{m} \mathrm{Cu}$ layer had been completely consumed during the assembly of the joints. The reason for the formation of
$\left(\mathrm{Cu}_{1-\mathrm{x}} \mathrm{Ni}_{\mathrm{x}}\right)_{6} \mathrm{Sn}_{5}$, instead of $\mathrm{Ni}_{3} \mathrm{Sn}_{4}$, on the substrate side can be found in the literature. ${ }^{14-16}$ It is mainly because the presence of a small amount of $\mathrm{Cu}$ has the ability to change the solder/ $\mathrm{Ni}$ reaction product from a $\mathrm{Ni}_{3} \mathrm{Sn}_{4}$-based compound to a $\mathrm{Cu}_{6} \mathrm{Sn}_{5}$-based one. As shown in Fig. $7 \mathrm{a}, \mathrm{AuSn}_{4}$ particles were clearly visible. When the original $0.65 \mu \mathrm{m} \mathrm{Au}$ of the surface finish dissolved completely into the solder, the effective Au concentration became 1.45 wt.\%, which was a value favorable for the formation of $\mathrm{AuSn}_{4} \cdot{ }^{16}$ After $300 \mathrm{~h}$ current stressing, as shown in Fig. $7 \mathrm{~b}$, the $\mathrm{Cu}_{6} \mathrm{Sn}_{5}$ near the upper-right corner of the joint had become $\left(\mathrm{Cu}_{1-\mathrm{x}} \mathrm{Ni}_{\mathrm{x}}\right)_{6} \mathrm{Sn}_{5}$, but for those $\mathrm{Cu}_{6} \mathrm{Sn}_{5}$ elsewhere over the UBM, no $\mathrm{Ni}$ can be detected. After $550 \mathrm{~h}$ of current stressing, as shown in Fig. 7c, on the substrate side, a nearly continuous layer of $\left(\mathrm{Au}_{1-\mathrm{y}} \mathrm{Ni}_{\mathrm{y}}\right) \mathrm{Sn}_{4}$ had formed over the $\left(\mathrm{Cu}_{1-\mathrm{x}} \mathrm{Ni}_{\mathrm{x}}\right)_{6} \mathrm{Sn}_{5}$ layer. In the literature, $\left(\mathrm{Au}_{1-\mathrm{y}} \mathrm{Ni}_{\mathrm{y}}\right) \mathrm{Sn}_{4}$ had been reported to form over the $\mathrm{Ni}_{3} \mathrm{Sn}_{4}$ layer after aging in order to have itself saturated with $\mathrm{Ni}^{16}$ The result of this study shows that $\left(\mathrm{Au}_{1-\mathrm{y}} \mathrm{Ni}_{\mathrm{y}}\right) \mathrm{Sn}_{4}$ can also form over the $\left(\mathrm{Cu}_{1-\mathrm{x}} \mathrm{Ni}_{\mathrm{x}}\right)_{6} \mathrm{Sn}_{5}$ layer in order to yield Ni. The fine-grain microstructure in Fig. 7d shows that the joint had failed through melting when the current stressing time reached $600 \mathrm{~h}$.

Shown in Fig. 8a is the zoom-in micrograph of the UBM region of Fig. 7b. According to the condition of the $\mathrm{Ni}$ layer, three regions can be identified from right to left: the two-phase region, the region in which $\mathrm{Ni}$ had been exhausted, and the region in which some $\mathrm{Ni}$ remained. With further current stressing, more $\mathrm{Ni}$ was consumed, as shown in Fig. 8b. The amount of 

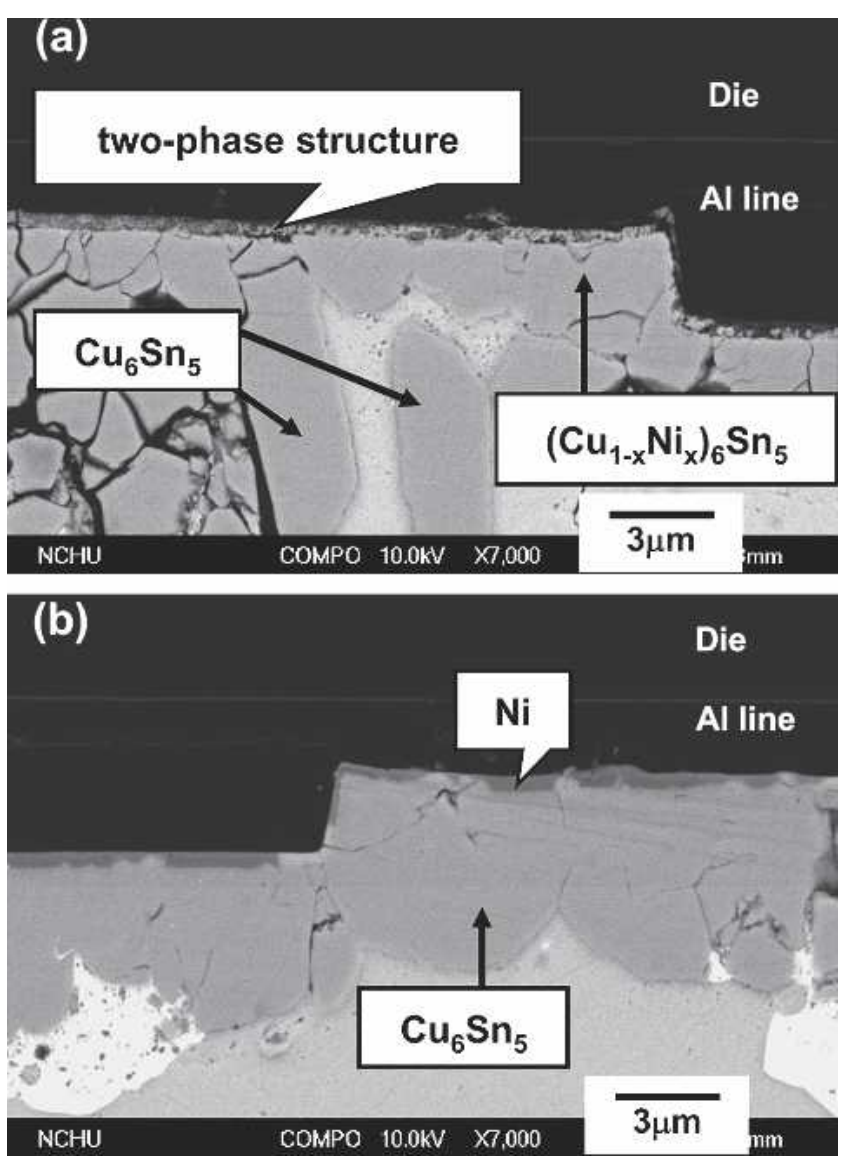

Fig. 3. Micrographs showing (a) the upper-right corner and (b) the upper-left corner of Fig. 2a. The sample was an OSP joint that had been stressed for $700 \mathrm{~h}$.

the two-phase structure and the Ni-exhausted region had increased, but only a small region to the far left still had unconsumed Ni. In short, the Ni consumption was asymmetric. The Ni UBM was depleted first on the right side, and then the depletion region propagated toward the left with increasing current stressing.

\section{DISCUSSION}

The most important finding of this study is that OSP solder joints had a MTTF value six times greater than that of the $\mathrm{Au} / \mathrm{Ni}$ joints. From the comparison of the microstructure evolution presented in the previous section, one can conclude that the main reason was that the Ni UBM consumption rate for the OSP joint was lower. In the following, we will (1) briefly summarize the failure mechanism for the $\mathrm{Au} /$ $\mathrm{Ni}$ solder joints, (2) explain why OSP joints had a lower $\mathrm{Ni}$ consumption rate, and (3) briefly summarize the failure mechanism for the OSP solder joints.

\section{Failure Mechanism for the Au/Ni Joints:}

\section{Accelerated Local Ni Consumption Due} to High Local Current Density and Local Joule Heating

Electromigration in flip-chip solder joints can cause failures through a void formation-and-propagation

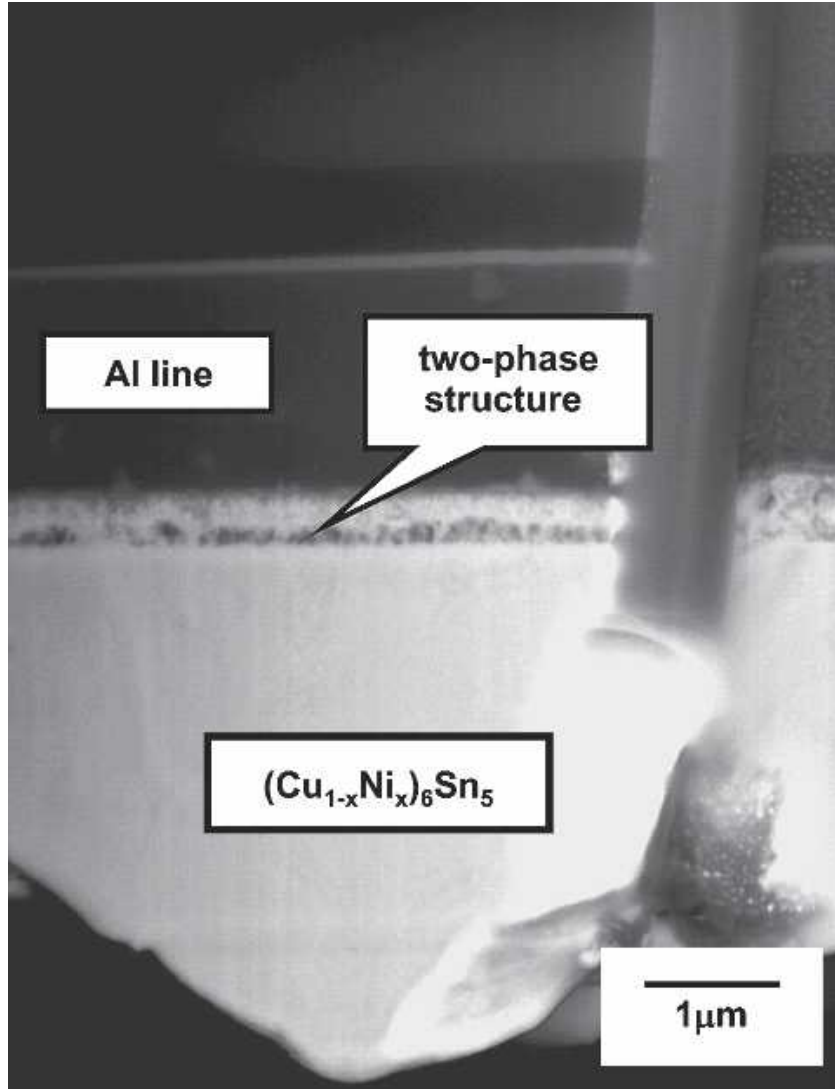

Fig. 4. FIB treated surface of the two-phase structure shown in Fig. 3a.

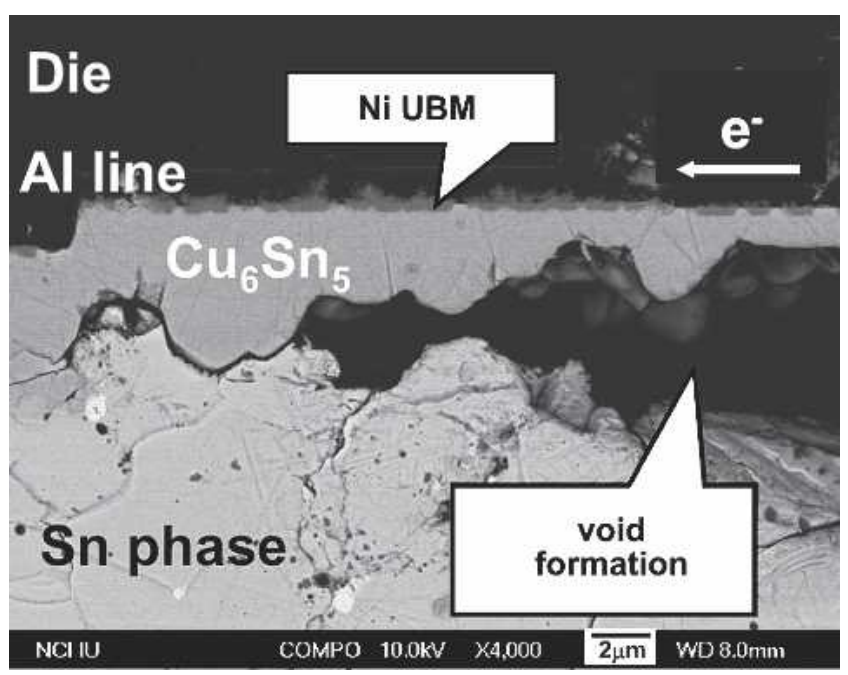

Fig. 5. Zoom-in view for the left side of the void shown in Fig. 2b. This figure shows that the void could continue to propagate to the left as long as the Ni UBM above had not been exhausted.

mechanism. ${ }^{2,4}$ In this mechanism, a void nucleates in the solder near the entrance of electrons from the interconnect. At this location, a high degree of current crowding occurs because the cross-sectional area of the interconnect is one to two orders of magnitude smaller than that of the solder joint. Once the void is nucleated, the region becomes nonconductive, and the electrons are diverted to the neighboring region of the void. This region, in turn, 

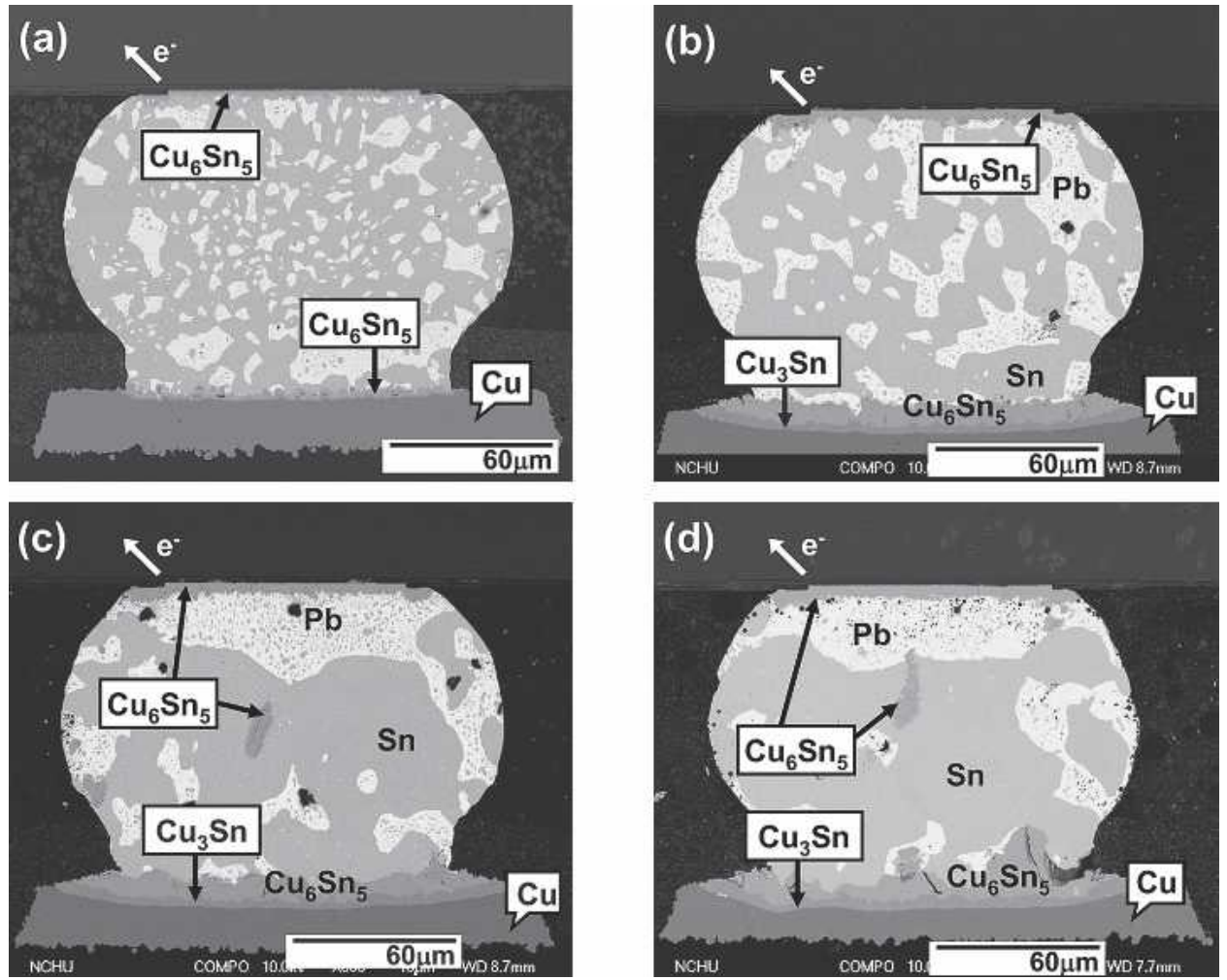

Fig. 6. Microstructures of the OSP joints that had been stressed at $150^{\circ} \mathrm{C}$ for (a) $0 \mathrm{~h}$, (b) $300 \mathrm{~h}$, (c) $700 \mathrm{~h}$, and (d) $1,700 \mathrm{~h}$. The electrons entered the joints from the substrates and left the joints through the chip.
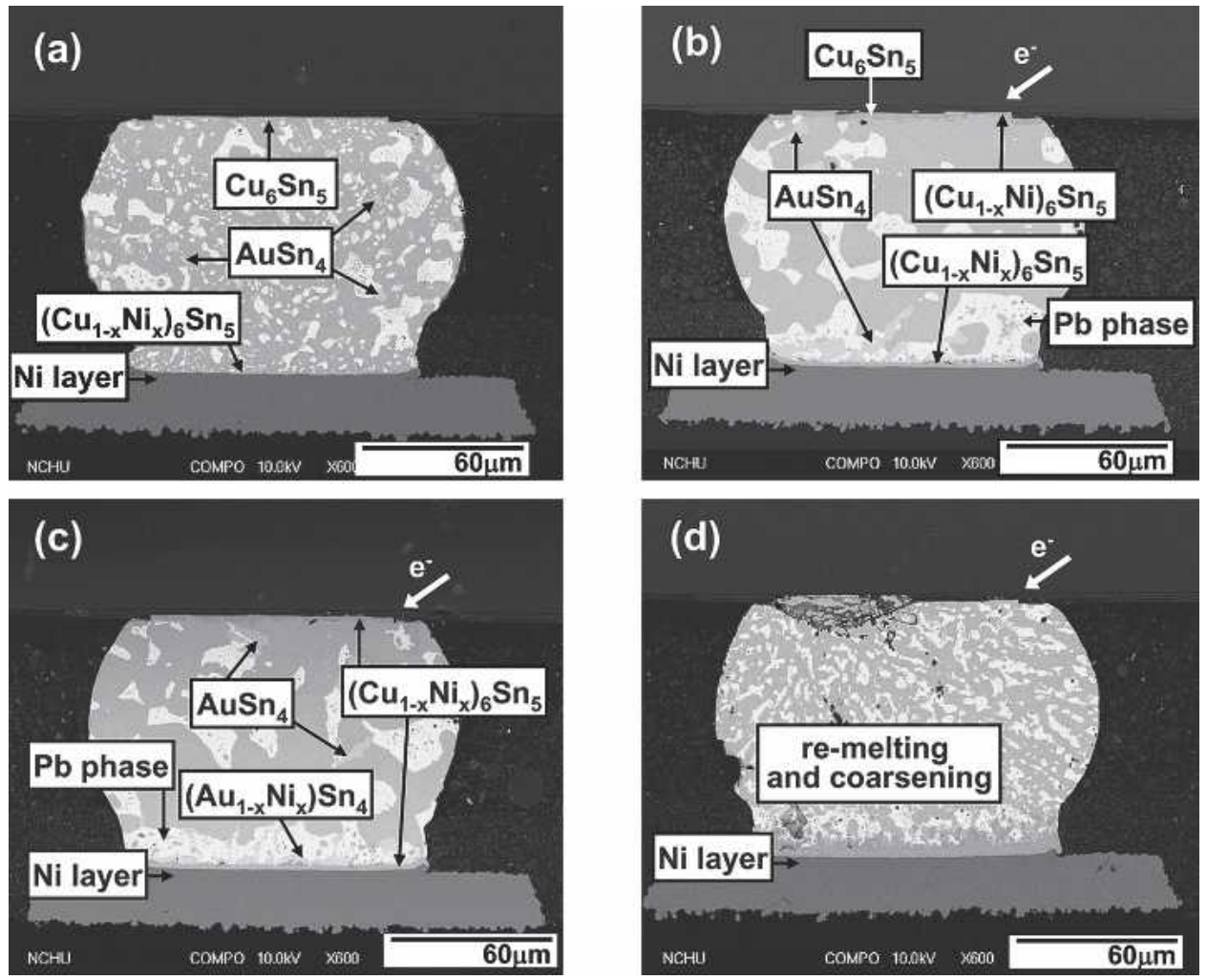

Fig. 7. Microstructures of the Au/Ni joints that had been stressed at $150^{\circ} \mathrm{C}$ for (a) $0 \mathrm{~h}$, (b) $300 \mathrm{~h}$, (c) $550 \mathrm{~h}$, and (d) $600 \mathrm{~h}$. The electrons entered the joints from the upper-right corners. 


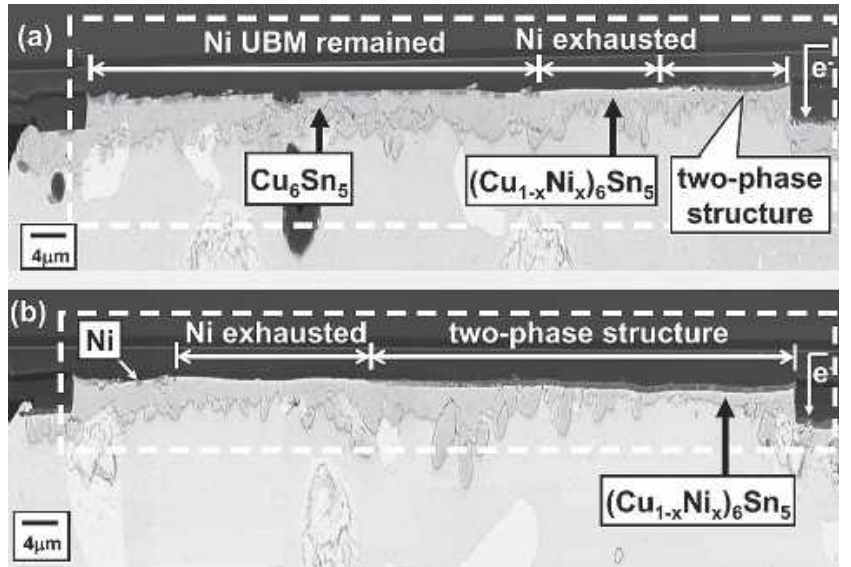

Fig. 8. (a) Zoom-in micrograph for the UBM region of Fig. 7b. The sample was an Au/Ni joint that had been stressed for $300 \mathrm{~h}$. (b) Zoom-in micrograph for the UBM region of Fig. 7c. The sample was an Au/Ni joint that had been stressed for $550 \mathrm{~h}$.

becomes the high current crowding region, and the void spreads quickly over. This process repeats itself and eventually causes the joint to fail. In the $\mathrm{Au} / \mathrm{Ni}$ joint used in this study, the high current crowding did not result in the formation of the void, but it induced an accelerated local Ni UBM consumption. Once $\mathrm{Ni}$ was completely consumed at a certain location, a nonconductive two-phase structure formed. The flow of the electrons was then diverted to the neighboring region. This neighboring region then became the place where electrons entered the joint, and the local Ni UBM was consumed at an accelerated rate. This process repeated itself, and the Nidepleted region extended farther on, creating an ever larger nonconductive region. The joint eventually failed through melting when a high percentage of the contact window was no longer conductive. In this study, the local Ni UBM was completely consumed before a void could nucleate in the solder. It had been reported that electromigration induced void nucleation needed an incubation period. ${ }^{2,12}$ There was a competition between this void incubation period and the time required to form the twophase structure. If the incubation period was longer than the time required to form the two-phase structure, the two-phase structure replaced the void as the role responsible for diverting the electron flow. If a thicker Ni UBM had been used, the void could have nucleated before the $\mathrm{Ni}$ was completely consumed. In such a situation, the void formationand-propagation mechanism would then become the failure mechanism.

The accelerated local $\mathrm{Ni}$ consumption could be ascribed to the combined effect of the high local current density and the resulting local Joule heating. The local current crowding area had a high current density, which enhanced the local diffusion of $\mathrm{Ni}$ away from the Ni UBM. This was the first factor responsible for the accelerated local $\mathrm{Ni}$ consumption. In addition, the local high current density also produced a high local Joule heating. As a result, the local area of the Ni UBM where the electrons entered the solder joint experienced the highest temperature. Therefore, the local Ni consumption was accelerated due to the local high temperature. The local accelerated Ni consumption also revealed itself through the observation that $\mathrm{Cu}_{6} \mathrm{Sn}_{5}$ had been converted locally to $\left(\mathrm{Cu}_{1-\mathrm{x}} \mathrm{Ni}_{\mathrm{x}}\right)_{6} \mathrm{Sn}_{5}$, as shown in Fig. 8 .

\section{Au/Ni vs. OSP Surface Finish}

In the OSP case, the intermetallic compound formed at the solder/Ni-UBM interface was always a $\mathrm{Cu}_{6} \mathrm{Sn}_{5}$-based compound. The $\mathrm{Ni}_{3} \mathrm{Sn}_{4}$ compound did not form because there was $30 \mu \mathrm{m} \mathrm{Cu}$ on the substrate side that served as an infinite $\mathrm{Cu}$ source. In addition, the $0.8-\mu \mathrm{m} \mathrm{Cu}$ layer existed over the Ni UBM initially. During assembly, this $\mathrm{Cu}$ layer dissolved completely into the solder, and contributed to the formation of a high initial $\mathrm{Cu}$ concentration which inhibited the formation of $\mathrm{Ni}_{3} \mathrm{Sn}_{4}$. Under such a condition, $\mathrm{Ni}_{3} \mathrm{Sn}_{4}$ could not form due to thermodynamic reasons. ${ }^{16,17}$ In contrast, the $\mathrm{Cu}$ source for the $\mathrm{Au} / \mathrm{Ni}$ joints was limited to the initial $0.8-\mu \mathrm{m} \mathrm{Cu}$ layer. The $30-\mu \mathrm{m} \mathrm{Cu}$ layer on the substrate side was blocked by the Ni layer of the $\mathrm{Au} / \mathrm{Ni}$ surface finish. As reported in the literature ${ }^{15,16}$ the infinite $\mathrm{Cu}$ source of the OSP joints could reduce the Ni UBM consumption rate through the so-called cross-interaction effect or simply the chemical effect. ${ }^{12}$ Comparing the results of $\mathrm{Au} / \mathrm{Ni}$ case and the OSP case, we noted that the former had a much faster Ni UBM consumption than the latter. This is the reason the OSP surface finish had a much longer MTTF.

A comparison between Fig. $2 \mathrm{a}-2 \mathrm{~b}$ and Fig. $6 \mathrm{c}-6 \mathrm{~d}$ also reveals the combined effect of the electromigration $\mathrm{Cu}$ flux and the cross-interaction $\mathrm{Cu}$ flux. In Fig. 2a and 2b, the electromigration tended to drive $\mathrm{Cu}$ atoms downward, but the cross-interaction or the chemical driving force tended to drive $\mathrm{Cu}$ upward. Conversely, in Fig. 6c and 6d, the electromigration $\mathrm{Cu}$ flux and the cross-interaction $\mathrm{Cu}$ flux were in the same direction (upward). In Fig. 6d, the $\mathrm{Cu}_{6} \mathrm{Sn}_{5}$ layer on the substrate side was thinner compared to that in Fig. 2b. In Fig. 6 d, $\mathrm{Cu}_{6} \mathrm{Sn}_{5}$ particles were even located near the center of the joints. This was caused by the fact that both the electromigration force and the cross-interaction force drove $\mathrm{Cu}$ atoms upward. In Fig. 2, the electromigration force and the cross-interaction force were in the opposite direction, and less $\mathrm{Cu}$ was driven upward, resulting in a thicker $\mathrm{Cu}_{6} \mathrm{Sn}_{5}$ on the substrate side.

\section{Failure Mechanism for the OSP Joints}

In the OSP solder joints, the Ni consumption rate was reduced due to the infinite $\mathrm{Cu}$ supply from the substrate side. As a result, the propagation of the two-phase structure from right to left in Fig. 2 was much slower. This reduced propagation rate provided the opportunity for the void to nucleate. For the OSP joints, the early sequence of events was the same as that of the $\mathrm{Au} / \mathrm{Ni}$ joints but at a reduced 
rate. When the overall conducting cross-section area decreased due to the propagation of the two-phase structure, the average current density increased. The higher average current density caused the incubation time for the void nucleation to decrease, and a void was able to nucleate before the Ni UBM immediately above the nucleation site being consumed completely. This was indeed what can be observed in Fig. 5, which shows that the Ni UBM above the void was still intact. The void could continue propagating to the left as long as the Ni UBM above had not been exhausted.

\section{CONCLUSIONS}

A key finding from this work is that the joints with the OSP surface finish had a MTTF six times greater than those with the $\mathrm{Au} / \mathrm{Ni}$ surface finish. The evolution of the microstructure led us to conclude that the OSP joints had a longer MTTF because these joint had a slower Ni UBM consumption rate. The lower consumption rate was due to the $\mathrm{Cu}-\mathrm{Ni}$ cross-interaction effect that had been reported in the literature. ${ }^{15,16}$

This study also shows that the combined effect of higher local current density and the resulting local Joule heating was able to produce accelerated local $\mathrm{Ni}$ consumption. Once the Ni UBM was completely consumed at a certain location, a two-phase structure formed. This two-phase structure was presumably nonconductive, and the flow of electrons was diverted to the neighboring region. In other words, the role played by the two-phase structure was quite similar to the role of void in the void formation-and-propagation mechanism reported in the literature. ${ }^{2,4}$ The void formation-and-propagation mechanism and the Ni UBM local consumption mechanism reported in this study were in competition as both the void and the porous structure were nonconductive and were able to divert the electrons.

\section{ACKNOWLEDGEMENT}

This work was supported by the National Science Council of Taiwan, Republic of China, through Grant Nos. NSC-94-2216-E-008-001 and NSC-942214-E-008-005.

\section{REFERENCES}

1. C.Y. Liu, K.N. Tu, T.T. Sheng, C.H. Tung, D.R. Frear, and P. Elenius, J. Appl. Phys. 87, 750 (2000).

2. E.C.C. Yeh, W.J. Choi, K.N. Tu, P. Elenius, and H. Balkan, Appl. Phys. Lett. 80, 580 (2002).

3. Y.C. Hu, Y.H. Lin, C.R. Kao, and K.N. Tu, J. Mater. Res. 18, 2544 (2003).

4. W.J. Choi, E.C.C. Yeh, and K.N. Tu, J. Appl. Phys. 94, 5665 (2003).

5. Y.C. Hsu, T.L. Shao, C.J. Yang, and C. Chen, J. Electron. Mater. 32, 1222 (2003).

6. H. Ye, C. Basaran, and D. Hopkins, Appl. Phys. Lett. 82, 1045 (2003).

7. T.L. Shao, Y.H. Chen, S.H. Chiu, and C. Chen, J. Appl. Phys. 96, 4518 (2004).

8. T.L. Shao, S.W. Liang, T.C. Lin, and C. Chen, J. Appl. Phys. 98, 044509 (2005)

9. J.W. Nah, J.O. Suh, and K.N. Tu, J. Appl. Phys. 98, 013715 (2005).

10. Y.H. Lin, C.M. Tsai, Y.C. Hu, Y.L. Lin, and C.R. Kao, J. Electron. Mater. 34, 27 (2005).

11. H. Gan and K.N. Tu, J. Appl. Phys. 97, 063514 (2005).

12. Y.H. Lin, Y.C. Hu, C.M. Tsai, C.R. Kao, and K.N. Tu, Acta Mater. 53, 2029 (2005)

13. C.M. Tsai, Y.L. Lin, J.Y. Tsai, and C.R. Kao, J. Electron. Mater. 35, 1005 (2006).

14. Y.L. Lin, C.W. Chang, C.M. Tsai, and C.R. Kao, J. Electron. Mater. 35, 1010 (2006).

15. C.M. Tsai, W.C. Luo, C.W. Chang, Y.C. Shieh, and C.R. Kao, J. Electron. Mater. 33, 1424 (2004).

16. C.E. Ho, S.C. Yang, and C.R. Kao, J. Electron. Mater. 31, 1264 (2002).

17. L.C. Shiau, C.E. Ho, and C.R. Kao, Solder. Surf. Mt. Tech. 14, 25 (2002). 